\title{
Functional Outcome of Intra-articular Fractures of Distal Radius: ORIF with Locking Plate vs CRPP
}

\author{
Shrestha $\mathrm{B}^{1^{*}}$, Sapkota $\mathrm{K}^{2}$, Kandel ${ }^{1}$, Dhakal $\mathbf{R M}^{3}$, Bista $\mathrm{K}^{3}$ \\ ${ }^{1}$ Associate Professor \& HOD, ${ }^{3}$ Lecturer, Department of Orthopedics, \\ Gandaki Medical College \& Teaching Hospital, Pokhara, Nepal \\ ${ }^{2}$ Assistant Professor, Manipal College of Medical sciences, Pokhara, Nepal
}

\author{
Keywords \\ CRPP, Distal fracture of radius, \\ Functional outcome, ORIF.

\section{Corresponding author} \\ ${ }^{*}$ Dr Bhola Shrestha \\ Associate Professor \& HOD \\ Department of Orthopedics \\ Gandaki Medical College \& Teaching \\ Hospital, Pokhara, Nepal \\ E-mail:bhola4355@hotmail.com
}

\begin{abstract}
Background: Distal radius fractures are common, costly, and increasing in incidence. Closed reduction and percutaneous $\mathrm{K}$ wires fixation and ORIF with locking plate fixation are two of the mostly used surgical treatments for dorsally displaced distal radius fractures. However, there is uncertainty which of these treatments is superior.
\end{abstract}

Methods: This is a prospective study on 40 patients. Twenty patients had closed reduction and percutaneous pinning (CRPP) with K wires (Group A) and 20 patients had open reduction and internal fixation (ORIF) with locking plate and screws (Group B). Evaluation were done in the means of DASH scores and ROM of operated wrist on 1.5 months, three months and six months follow up period.

Results: Though the overall functional outcome is comparatively better with Group B, the significant difference for most variables like DASH Scores, extension, supination and ulnar deviation were found at early part of treatment only. The flexion and radial deviation bear no significant outcomes at all intervals of evaluations. Superficial wound infection was more common in patients treated with $\mathrm{K}$-wires but otherwise no difference in complication rates was noted.

Conclusion: Locking plate fixation provided lower DASH scores and reduced total postoperative complications compared to CRPP group over six months follow-up period. However, these differences were significant in early part of treatment. Further research is required to better delineate the confirmation.

\section{INTRODUCTION}

Fractures of distal part of the radius are common and account for approximately $15 \%$ of all of the fractures in adults $^{1}$. Dispalced intra-articular distal radial fractures continue to pose challenges with regard to both decision making and management when treating wrist injuries. Striving for an anatomic reduction of the articular surface is accepted as a desirable goal because articular incongruities after fracture healing have been documented to adversely affect functional outcome and lead to early degenerative changes ${ }^{2}$.
Most fractures of the distal radius are reducible with adequate stability and can be treated by closed reduction and casting. However, fractures that are unstable or involve the articular surfaces can jeopardize the congruence and kinetics of the wrist ${ }^{3}$. In complex intra-articular fractures of the distal radius, surgery allows more accurate reduction of articular surface and treatment of soft tissue injuries ${ }^{4}$.

We undertook this prospective study of intra-articular fractures of the distal radius to evaluate functional outcomes after reduction either by surgery (Open reduction and internal fixation with locking plate) or by 
closed reduction and per cutaneous fixation with K-wires under fluoroscopy.

\section{METHODS}

A total of 40 consecutive patients, 18 females, 24 males, treated between December 1, 2015 and December 1, 2016 (15 Mangsir 2072 to 16 Mangsir 2073 B.S.) were included in the study. The study was conducted in Fishtail Hospital, Pokhara and Gandaki Medical College Teaching Hospital, Pokhara. They were assigned, based on convenient sampling for treatment. A written informed consent was obtained from each patient. Approval from the institutional review board was granted.

All patients had similar fracture characteristics. Those admitted to the study had an intra-articular fracture of distal radius (AO type- C1, C2 or C3) with a step off or gap greater than $2 \mathrm{~mm}$. Patients with open fractures, multiple fractures, or associated with others upper extremity injuries were excluded. Patients with central nervous system disorders such as previous stroke or cerebral palsy were also excluded.

Twenty patients had closed reduction and percutaneous pinning (CRPP) with K-wires (Group A). This group included 10 males and 10 females with a mean age of 51 years (44 to 66 years). Twenty patients had open reduction and internal fixation (ORIF) with locking plate and screws (Group B). There were 12 males and 8 females with a mean age of 41 years ( 30 to 52 years) in Group B. The mechanism of injury was a fall on outstretched extremity in 30 patients and a motor vehicle accident in 10 . The operations were performed under general or regional anesthesia.

In all patients of Group A, cast and K-wires were removed on first post operative month and asked to do exercise at home. In Group B, sutures were removed in interval of 10 to 14 post-operative days and asked to do exercises at home. All patients were requested to follow up on 1.5 months, three months and six months.

At 1.5 months, three months and six months postoperatively, all patients were examined using the Disabilities of the Arm, Shoulder and Hand (DASH) scores questionnaire and range of motion of operated wrist like Flexion (FX), Extension (EX), Suppination (SU), Pronation (PR), Ulnar deviation (UD) and Radial deviation (RD). Antero-posterior and lateral radiograph of the distal radius were done during the diagnosis and in each follow up period.

Global hand function was evaluated using the upper limb functional evaluation scoring system-the DASH score. The DASH is a validated 30-item, self-report questionnaire designed to measure physical function and symptoms in patients with musculoskeletal disorders of the upper limb, with a total score ranging between ' 0 ', indicative of normal use of the upper limb, to '100', indicative of a nonfunctional upper limb.

All data were analyzed by SPSS 17.0 and descriptive statistics were applied. Significant of the means were compared by using $\mathrm{T}$ test. The results were considered to be significant with p-value of $<0.05$.

\section{RESULTS}

Group A have DASH Scores of $75.4 \pm 3.89$ in 1.5 months follow up where as Group B have DASH scores of 26.2 \pm 2.75 in same duration. Group B, comparatively has less DASH Scores than Group A at all follow up evaluations as shown in Table 1. But the significant difference is found to be at 1.5 months interval with $\mathrm{p}$ value $<0.05$. However, over all period of time, there is gradual reduction in DASH Scores in both Groups.

There is significantly different in flexion of wrist of Group A and Group B in 1.5 months follow up. But, in six months follow up, flexion of wrist of Group A is $61.1 \pm 1.20$ and flexion of wrist of Group B is $64.7 \pm 1.49$. But extension of wrist of Group A is $4.95 \pm 1.6$ and that of Group B is $28.15 \pm 2.90$ in 1.5 months follow up, which is statistically significant ( $p$ value $<0.05$ ). Whereas the extension of wrist of Group A is $52.6 \pm 2.50$ and extension of wrist of Group B is $64 \pm 2.95$ in six months follow up (Table 3). Supination and pronation in both groups have significantly different in 1.5 months and three months follow up ( $p$ value $<0.05$ ) but there is no such different in six months follow up, as shown in Table 4 and 5.

In 1.5 months follow up, radial deviation of Group $\mathrm{A}$ is $3.45 \pm 0.82$ and $5.2 \pm 0.95$ in Group B. It gradually increased to $8.45 \pm 0.51$ in Group A and $9.3 \pm 0.47$ in Group B in six months follow up (Table 6). Ulnar deviation in Group A is $4.2 \pm 0.83$ and ulnar deviation in Group B is $5.7 \pm 0.57$ in 1.5 months follow up, which is statistically significant ( $p$ value $<0.05$ ).

Complications were encountered in two patients. In Group 
A, two patients had superficial pin tract infection around a K-wire. This was resolved by oral antibiotics and early removal of the pins was done.

Table 1: DASH scores in each follow up

\begin{tabular}{lccc} 
& DASH $1 \pm$ SD & DASH $2 \pm$ SD & DASH $3 \pm$ SD \\
Group A & $75.4 \pm$ & $36.4 \pm$ & $27.1 \pm$ \\
Group B & $26.2 \pm$ & $21.4 \pm$ & $17.4 \pm$ \\
P value & 0.04 & 0.01 & 0.57 \\
\hline
\end{tabular}

Note: $1=1.5$ months follow up; $2=3$ months follow up;

$3=6$ months follow up

Table 2: Degree of flexion in each follow up

\begin{tabular}{lccc} 
& Flexion $\mathbf{1} \pm \mathbf{S D}$ & Flexion $\mathbf{2} \pm \mathbf{S D}$ & Flexion $\mathbf{3} \pm \mathbf{S D}$ \\
Group A & $8.45 \pm 1.66$ & $49.2 \pm 3.91$ & $61.1 \pm 1.2$ \\
Group B & $31.2 \pm 1.5$ & $54.15 \pm 1.49$ & $64.7 \pm 1.49$ \\
P value & 0.75 & 0.1 & 0.26 \\
\hline
\end{tabular}

Table 3: Degree of extension in each follow up

\begin{tabular}{lccc}
\hline & Extension $\mathbf{1} \pm$ SD & Extension $2 \pm$ SD & Extension $3 \pm$ SD \\
Group A & $4.95 \pm 1.6$ & $13.75 \pm 3.1$ & $52.6 \pm 2.5$ \\
Group B & $28.15 \pm 2.9$ & $42 \pm 3.29$ & $64 \pm 2.95$ \\
P value & 0.012 & 0.05 & 0.88 \\
\hline
\end{tabular}

Table 4: Degree of supination in each follow up

\begin{tabular}{lccc}
\hline & Supination $\mathbf{1} \pm$ SD & Supination $2 \pm$ SD & Supination $3 \pm$ SD \\
Group A & $9.1 \pm 3.2$ & $33.55 \pm 5.4$ & $68.1 \pm 3.69$ \\
Group B & $23.15 \pm 3.29$ & $50.3 \pm 4.06$ & $71.9 \pm 1.65$ \\
P value & 0.041 & 0.02 & 0.075 \\
\hline
\end{tabular}

Table 5: Degree of pronation in each follow up

\begin{tabular}{lccc} 
& Pronation $\mathbf{1} \pm$ SD & Pronation $2 \pm$ SD & Pronation $3 \pm$ SD \\
Group A & $9.45 \pm 3.05$ & $35.15 \pm 6.51$ & $76.45 \pm 3.48$ \\
Group B & $24.35 \pm 3.26$ & $51 \pm 3.74$ & $78.75 \pm 1.40$ \\
P value & 0.015 & 0.003 & 0.015 \\
\hline
\end{tabular}

Table 6: Degree of radial deviation in each follow up

\begin{tabular}{llll}
\hline & Radial Deviation $1 \pm$ SD & Radial Deviation $2 \pm$ SD & Radial Deviation $3 \pm$ SD \\
Group A & $3.45 \pm 0.82$ & $6.45 \pm 0.68$ & $8.45 \pm 0.51$ \\
Group B & $5.2 \pm 0.95$ & $7.85 \pm 0.67$ & $9.3 \pm 0.47$ \\
P value & 0.13 & 0.41 & 0.09 \\
\hline
\end{tabular}

Table 7: Degree of ulnar deviation in each follow up

\begin{tabular}{lccc}
\hline & Ulnar Deviation $1 \pm$ SD & Ulnar Deviation $2 \pm$ SD & Ulnar Deviation $3 \pm$ SD \\
Group A & $4.2 \pm 0.83$ & $15.05 \pm 1.57$ & $19.2 \pm 1.36$ \\
Group B & $5.7 \pm 0.57$ & $19.3 \pm 1.17$ & $20.7 \pm 0.97$ \\
P Value & 0.03 & 0.55 & 0.24 \\
\hline
\end{tabular}

\section{DISCUSSION}

Open reduction and internal fixation of fractures of the distal radius allows direct observation of articular surfaces. In particular, rotation of the fracture fragments, which is difficult to judge under fluoroscopy, may be detected and corrected on surgery. Irrigation to remove fracture hematoma and debris potentially reduces the inflammatory reaction and improves the range of movement ${ }^{4}$. Open surgery also allows for the management of associated acute soft-tissue injuries, whose treatment has a better prognosis than chronic lesions. Our study confirms this observation, as the patients in the ORIF group showed better results after immediate treatment of acute soft tissue injuries, not withstanding reports showing a discrepancy between radiological appearance and function ${ }^{5}$.

Treatment of displaced fractures of the distal radius aims to restore normal anatomy ${ }^{6}$. An intra-articular step of more than $2 \mathrm{~mm}$ will not be generally accepted ${ }^{7}$, and recent studies indicate that the critical tolerance may be as low as $1 \mathrm{~mm}^{8}$. Some advocate for computed tomography scan for articular incongruity in this fracture, but it is not possible to do computed tomography in all the cases. Johnston et $a l^{9}$ described their experience with computed tomography scanning in determining operative indications and preoperative planning for distal radial fractures, but their study did not determine if a computed tomography scan is better than or equivalent to radiographs in detecting fracture displacements and combination.

The incidence of associated ligament injuries had been variously reported: lesions of the scapholunate ligament in up to $40 \%$ of intra-articular fractures, lunotriquetral ligament injuries in $20 \%$, and combined scapholunate and lunotriquetral injuries in $10 \%$ of fractures ${ }^{10}$. Finally, articular cartilage lesions have been noted in both the radiocarpal and midcarpal joints in up to $30 \%$ of distal radial fractures in young adults ${ }^{11}$.

The operation time was significantly increased by approximately 45 minutes in the ORIF group, but there is no other study for comparison. There were also differences in hospital stay or time to mobilization in two groups. Although the range of movement was acceptable in both groups, this study shows the ORIF technique to be superior, at least in the early months. There was a statistically significant difference between the outcomes 
in the two groups which decreased with time.

In our study, there was no correlation between the outcome score and type of fracture, age, gender or operative delay. Slight loss of movement and grip strength was the main reason for inferior results. All patients returned to their regular activities but this occurred earlier in the Group B patients. Differences regarding return to work were not statistically significant. In Group A, patients returned to work after a mean of four months (Three to five months) where as in Group B, patients returned to work after a mean of 2.5 months (Two to three months).

DASH scores tend to show similarity at the end of six months. No significant difference existed in DASH scores at six months in many recent studies ${ }^{12}$. DASH scores were significantly lower (i.e. less impairment in upper limb function), for all time points of measurement over six months postoperatively, for patients who underwent ORIF with locking plate compared to patients with CRPP. The difference in DASH scores between the two groups, however, decreased over the time. It is possible that the higher DASH scores for patients with CRPP over the initial postoperative phase may be due to delayed onset of wrist ROM exercises. Therefore, the locking plate fixation technique could be considered for patients requiring a faster return to function after injury.

We found range of wrist flexion, supination and pronation to be significantly better in patients with locking plate fixation at three and six months, again with no significant difference, compared to patients with CRPP at six months. There were no differences for other ROMs of the wrist between the two patient groups. In our study, we presumed that patient-reported functionally satisfaction, as recorded by the DASH scores, was partially related to objective assessments of wrist and hand function (i.e. ROM and daily activities), which could explain statistical differences in ROM between the two patient groups over the early postoperative period. But the ROMs of the wrist and DASH scores between the two patient groups are also similar at six months. Even extension, radial deviation and ulnar deviation show difference at six months. The argument of improved ROMs at an earlier time is not entirely true. So the selective return of wrist movements may not benefit the patient much clinically. We did not include radiographic data in our study because the radiographic parameters did not necessarily correlate with subjective functional outcomes ${ }^{13}$.
According to the result of our study, ORIF with locking plate may be a superior fixation technique, over a CRPP technique, for the distal radius fracture. However, from a cost-analysis perspective, the cost of locking plate to be two to threefold higher than that of K-wire fixation. Hence, surgeons must weigh all evidence while determining the treatment options for distal radius fractures in collaboration with the patient. The surgeon must provide patients with evidence-based information regarding the risks and benefits of the two surgical fixation techniques, taking into consideration a patient's expectations, lifestyle and associated injuries in determining the most appropriate treatment approach.

This study has several clinical limitations. We had a follow up on 1.5 months, three months and six months post operatively. Although an average follow-up of six months is sufficient to evaluate the result of fracture distal radius treatment, the results of long-term follow-up remain to be clarified. We had included only 40 cases in our study, which is very less in numbers to evaluate the outcome. The relatively small number of participants limited the statistical power of findings. Hence, future studies are needed to improve the statistical outcomes.

\section{CONCLUSION}

ORIF with locking plate fixation provided lower DASH scores and reduced total postoperative complications, most specifically lowering the risk for postoperative superficial infection compared to CRPP group over six months follow-up period. ORIF with locking plate fixation also provides better ROM of wrist in the six month postoperative period. However, owing to the limitation and bias of the evidence in our study, all the above viewpoints require larger and more rigorously powered multicentre studies for confirmation.

\section{REFERENCES}

1. Sanders WE. Distal radius fractures. In: Hand surgery update. Rosemont, IL: American Academy of Orthopaedic Surgery. 1996; P 117-23.

2. Kirk J, Jupiter B. Intra articular fractures of the distal end of the radius in young adults. $J$ Bone 
Joint Surg [Am]. 1986; 68: 647-59.

3. Grewal R, Perey B, Wilmink M, Stothers K. A randomized prospective study on the treatment of intra-articular distal radius fractures: Open reduction and internal fixation with dorsal plating versus mini open reduction, percutaneous fixation, and external fixation. J Hand Surg [Am]. 2005; 30-A: 764-72.

4. Geissler WB. Intra-articular distal radius fractures: the role of arthroscopy? Hand Clin. 2005; 21: 40716.

5. Catalano LE 3rd, Cole RJ, Gelberman RH, et al. Displaced intra-articular fractures of the distal aspect of the radius: Long term results in young adults after open reduction and internal fixation. $J$ Bone Joint Surg [Am]. 1997; 79-A: 1290-302.

6. Anderson DD, Bell AL, Gaffney MB, Imbriglia JE. Contact stress distributions in malreduced intraarticular distal radius fractures. J Orthop Trauma. 1996; 10: 331-7.

7. Viegas SF. Midcarpal arthroscopy: Anatomy and portals. Hand Clin. 1994; 10: 577-87.

8. Levy HJ, Glickel SZ. Arthroscopic assisted internal fixation of volar intraarticular wrist fractures. Arthroscopy. 1993; 47: 122-4.

9. Johnston GH, Freidman L, Kriegler JC. Computerized tomographic evaluation of acute distal radial fractures. J Hand Surg Am. 1992; 17: 738-44.

10. Richards RS, Bennett JD, Roth JH, Milne K. Arthroscopic diagnosis of intra-articular soft tissue injuries associated with distal radial fractures. $J$ Hand Surg [Am]. 1997; 22: 772-6.

11. Lindau T, Arner M, Hagberg L. Intraarticular lesions in distal fractures of the radius in young adults: a descriptive arthroscopic study in 50 patients. $J$ Hand Surg [Br]. 1997; 22: 638-43.

12. Karantana A, Downing ND, Forward DP, Hatton M, Taylor AM, Scammell BE, Moran CG, Davis TR. Surgical treatment of distal radial fractures with a volar locking plate versus conventional percutaneous methods: a randomized controlled trial. J Bone Joint Surg (Am Vol). 2013; 95(19): 1737-1744.

13. Arora R, Lutz M, Deml C, Krappinger D, Haug L, Gabl M. A prospective randomized trial comparing nonoperative treatment with volar locking plate fixation for displaced and unstable distal radial fractures in patients sixty-five years of age and older. J Bone Joint Surg (Am Vol). 2011; 93(23): 2146-2153. 